\title{
Synthesis of 3,4-Dihydropyrimidin-2(1H)-Ones and Their Corresponding 2(1H)Thiones Using Trichloroacetic Acid as a Catalyst under Solvent-Free Conditions
}

\author{
Zahed Karimi-Jaberi and Mohammad Sadegh Moaddeli \\ Department of Chemistry, Firoozabad Branch, Islamic Azad University, P.O. Box 74715-117, Firoozabad, Fars, Iran \\ Correspondence should be addressed to Zahed Karimi-Jaberi, zahed.karimi@yahoo.com \\ Received 3 August 2012; Accepted 8 October 2012 \\ Academic Editors: D.-M. Du and G. K. Patra
}

Copyright ( 2012 Z. Karimi-Jaberi and M. S. Moaddeli. This is an open access article distributed under the Creative Commons Attribution License, which permits unrestricted use, distribution, and reproduction in any medium, provided the original work is properly cited.

Trichloroacetic acid was found to be a convenient catalyst for the synthesis of 3,4-dihydropyrimidin-2-(1H)-ones and their corresponding $2(1 \mathrm{H})$-thiones through a one-pot three-component reaction of aldehydes, alkyl acetoacetate, and urea or thiourea at $70^{\circ} \mathrm{C}$ under solvent-free conditions.

\section{Introduction}

Biginelli reaction is a useful three-component reaction offering versatile protocol for the production of 3,4-dihydropyrimidin-2 $(1 \mathrm{H})$-ones which exhibit widespread biological applications such as antihypertensive, antiviral, antitumor, antibacterial, $\alpha$-1a-antagonism, antioxidant, and antiinflammatory actions $[9,10]$.

Although numerous catalysts have been developed in accelerating this reaction $[1-8,11-22]$, it is still desirable to develop this reaction using newer reagents with greater efficiency, simpler operational procedure, and milder reaction condition, and a higher yield of products coupled with potential bioactivity is important.

With the awareness of environmental issues and importance of this reaction and keeping our interest in the development of synthetic routes to heterocyclic compounds [23-27], herein, we report a heterogeneous, solid trichloroacetic acid, as an alternative, cheap, and efficient catalyst for the Biginelli reaction (Scheme 1).

Trichloroacetic acid is a readily available and inexpensive solid reagent and it has been used by our group for the synthesis of dihydropyrano[2,3-c]pyrazoles [23] and tetrahydrobenzo[a]xanthen-11-ones and dibenzo[a,j]xanthenes [24].

\section{Results and Discussion}

The catalytic activity of trichloroacetic acid was first investigated using three-component reaction of benzaldehyde, ethyl acetoacetate, and urea as a model reaction. After carrying out the reaction at different conditions, the best results have been obtained with $20 \mathrm{~mol} \%$ trichloroacetic acid at $70^{\circ} \mathrm{C}$ after $4 \mathrm{~min}$ with $85 \%$ yield under solvent-free conditions. In the absence of trichloroacetic acid, only $20 \%$ yield of the product was obtained even after heating at $70^{\circ} \mathrm{C}$ for $12 \mathrm{~h}$ with recovery of starting material.

The reaction was also examined in solvents such as $\mathrm{EtOH}, \mathrm{H}_{2} \mathrm{O}, \mathrm{CHCl}_{3}$, and toluene. In the presence of solvents, reaction was sluggish and the formation of by-products was observed. The reaction temperature was also optimized, below $70^{\circ} \mathrm{C}$ the reaction proceeded slow giving a relatively low yield and no improvement was observed above $70^{\circ} \mathrm{C}$.

Having established the reaction conditions, various 3,4dihydropyrimidin-2 $(1 \mathrm{H})$-ones were synthesized in excellent yields through the reaction of different aldehydes, alkyl acetoacetate, and urea. The results are summarized in Table 1, which clearly indicates the generality and scope of the reaction with respect to various aromatic, heteroaromatic, unsaturated, and aliphatic aldehydes. It is noteworthy that acid-sensitive aldehydes such as furfural and cinnamaldehyde 
TABLE 1: Trichloroacetic acid catalyzed one-pot synthesis of 3,4-dihydropyrimidin-2-(1H)-ones or thiones under solvent-free conditions.

\begin{tabular}{|c|c|c|c|c|c|c|c|}
\hline Entry & $\mathrm{R}_{1}$ & $\mathrm{R}_{2}$ & $\mathrm{X}$ & Time $(\min )$ & Yield (\%) & $\mathrm{mp}\left({ }^{\circ} \mathrm{C}, \mathrm{obsd}\right)$ & $\mathrm{mp}\left({ }^{\circ} \mathrm{C}\right.$, lit $)$ (ref.) \\
\hline 1 & $\mathrm{C}_{6} \mathrm{H}_{5}$ & OEt & $\mathrm{O}$ & 4 & 85 & $201-203$ & $202-205[1]$ \\
\hline 2 & $4-\mathrm{ClC}_{6} \mathrm{H}_{4}$ & OEt & $\mathrm{O}$ & 9 & 92 & $212-216$ & $210-212[1]$ \\
\hline 3 & $4-\mathrm{HOC}_{6} \mathrm{H}_{4}$ & OEt & $\mathrm{O}$ & 40 & 90 & $226-228$ & $231-233[2]$ \\
\hline 4 & $3-\mathrm{O}_{2} \mathrm{NC}_{6} \mathrm{H}_{4}$ & OEt & $\mathrm{O}$ & 20 & 93 & $225-228$ & $227-228[1]$ \\
\hline 5 & $4-\mathrm{O}_{2} \mathrm{NC}_{6} \mathrm{H}_{4}$ & OEt & $\mathrm{O}$ & 5 & 85 & $206-209$ & 207-209 [1] \\
\hline 6 & $\mathrm{C}_{6} \mathrm{H}_{5} \mathrm{CH}=\mathrm{CH}$ & OEt & $\mathrm{O}$ & 3 & 90 & $225-227$ & $223-226[1]$ \\
\hline 7 & $4-\mathrm{MeOC}_{6} \mathrm{H}_{4}$ & OEt & $\mathrm{O}$ & 20 & 95 & $200-202$ & $202-204[1]$ \\
\hline 8 & $2,4-(\mathrm{Cl})_{2} \mathrm{C}_{6} \mathrm{H}_{3}$ & OEt & $\mathrm{O}$ & 4 & 91 & $247-249$ & $246-248[3]$ \\
\hline 9 & 4- $\mathrm{MeC}_{6} \mathrm{H}_{4}$ & OEt & $\mathrm{O}$ & 5 & 90 & $213-215$ & $214-216[1]$ \\
\hline 10 & $2-\mathrm{MeOC}_{6} \mathrm{H}_{4}$ & OEt & $\mathrm{O}$ & 2 & 94 & $262-263$ & $260[4]$ \\
\hline 11 & $2,6-(\mathrm{Cl})_{2} \mathrm{C}_{6} \mathrm{H}_{3}$ & OEt & $\mathrm{O}$ & 3 & 96 & $226-228$ & $226[5]$ \\
\hline 12 & $2-\mathrm{ClC}_{6} \mathrm{H}_{4}$ & OEt & $\mathrm{O}$ & 9 & 85 & $221-223$ & $221-223[2]$ \\
\hline 13 & $4-\mathrm{BrC}_{6} \mathrm{H}_{4}$ & OEt & $\mathrm{O}$ & 11 & 90 & $212-214$ & $215[6]$ \\
\hline 14 & $\mathrm{CH}_{3}$ & OEt & $\mathrm{O}$ & 3 & 92 & $188-190$ & 194-195 [7] \\
\hline 15 & $\mathrm{CH}_{3} \mathrm{CH}_{2} \mathrm{CH}$ & OEt & $\mathrm{O}$ & 50 & 88 & $163-165$ & $164-166[7]$ \\
\hline 16 & $3-\mathrm{MeC}_{6} \mathrm{H}_{4}$ & OEt & $\mathrm{O}$ & 8 & 93 & $219-222$ & $224-226$ [2] \\
\hline 17 & 2-Furyl & OEt & $\mathrm{O}$ & 19 & 86 & $202-205$ & 202-204 [5] \\
\hline 18 & $\mathrm{C}_{6} \mathrm{H}_{5}$ & $\mathrm{OMe}$ & $\mathrm{O}$ & 5 & 94 & $208-211$ & $210-213[1]$ \\
\hline 19 & $4-\mathrm{MeOC}_{6} \mathrm{H}_{4}$ & $\mathrm{OMe}$ & $\mathrm{O}$ & 9 & 85 & $192-195$ & 193-196 [3] \\
\hline 20 & $4-\mathrm{ClC}_{6} \mathrm{H}_{4}$ & $\mathrm{OMe}$ & $\mathrm{O}$ & 8 & 92 & 204-206 & 203-205 [1] \\
\hline 21 & $4-\mathrm{O}_{2} \mathrm{NC}_{6} \mathrm{H}_{4}$ & $\mathrm{OMe}$ & $\mathrm{O}$ & 3 & 95 & $235-237$ & $235-236[5]$ \\
\hline 22 & $2-\mathrm{ClC}_{6} \mathrm{H}_{4}$ & $\mathrm{OMe}$ & $\mathrm{O}$ & 6 & 84 & $180-182$ & $181-183[1]$ \\
\hline 23 & $3-\mathrm{O}_{2} \mathrm{NC}_{6} \mathrm{H}_{4}$ & $\mathrm{OMe}$ & $\mathrm{O}$ & 12 & 90 & $271-274$ & $273-275[2]$ \\
\hline 24 & $4-\mathrm{MeC}_{6} \mathrm{H}_{4}$ & $\mathrm{OMe}$ & $\mathrm{O}$ & 14 & 93 & 206-209 & $210-213[3]$ \\
\hline 25 & $4-\mathrm{HOC}_{6} \mathrm{H}_{4}$ & $\mathrm{OMe}$ & $\mathrm{O}$ & 7 & 87 & $235-237$ & $231-233$ [2] \\
\hline 26 & $2-\mathrm{MeOC}_{6} \mathrm{H}_{4}$ & $\mathrm{OMe}$ & $\mathrm{O}$ & 2 & 95 & $284-286$ & $285-287$ [2] \\
\hline 27 & $3-\mathrm{MeC}_{6} \mathrm{H}_{4}$ & $\mathrm{OMe}$ & $\mathrm{O}$ & 4 & 96 & $214-217$ & $216-218$ [2] \\
\hline 28 & $3-\mathrm{ClC}_{6} \mathrm{H}_{4}$ & $\mathrm{OMe}$ & $\mathrm{O}$ & 9 & 92 & $208-211$ & $209-210$ [2] \\
\hline 29 & $2,4-(\mathrm{Cl})_{2} \mathrm{C}_{6} \mathrm{H}_{3}$ & $\mathrm{OMe}$ & $\mathrm{O}$ & 3 & 94 & $252-255$ & $252-253[3]$ \\
\hline 30 & 2-Furyl & $\mathrm{OMe}$ & $\mathrm{O}$ & 11 & 88 & $216-218$ & $214-216[8]$ \\
\hline 31 & $\mathrm{C}_{6} \mathrm{H}_{5}$ & OEt & $\mathrm{S}$ & 25 & 90 & $210-212$ & $210-212[1]$ \\
\hline 32 & $4-\mathrm{ClC}_{6} \mathrm{H}_{4}$ & OEt & $\mathrm{S}$ & 18 & 86 & $181-183$ & $184-185$ [3] \\
\hline 33 & $4-\mathrm{MeOC}_{6} \mathrm{H}_{4}$ & OEt & S & 20 & 85 & $136-138$ & $137-139$ [3] \\
\hline 34 & $3-\mathrm{O}_{2} \mathrm{NC}_{6} \mathrm{H}_{4}$ & OEt & $\mathrm{S}$ & 15 & 87 & 205-208 & 205-206 [8] \\
\hline 35 & $\mathrm{C}_{6} \mathrm{H}_{5}$ & $\mathrm{OMe}$ & $S$ & 13 & 92 & $220-222$ & $221-222$ [3] \\
\hline
\end{tabular}<smiles>[R]C(=O)C1=C(C)NC([X])NC1[R]</smiles>

Scheme 1

that (Table 1) worked well gave the corresponding products. The reaction can also proceed with methyl acetoacetate (Table 1, entries 18-30). In all cases, dihydropyrimidinones were the sole products and no by-product was observed.
The reaction of aldehydes with alkyl acetoacetate and thiourea under similar reaction conditions also provided the corresponding 3,4-dihydropyrimidin-2(1H)-thiones in high yields (Table 1, entries 31-35), which are also of interest with respect to their biological activities [21]. 


\section{Conclusion}

In conclusion, a novel approach to explore the use of trichloroacetic acid for the synthesis of 3,4-dihydropyrimidin-2- $(1 \mathrm{H})$-ones and their corresponding 2(1H)thione has been described through the Biginelli reaction at $70^{\circ} \mathrm{C}$ under solvent-free conditions. This method offers several advantages including high yields, short reaction times, solvent-free condition, a simple work-up procedure without using any chromatographic methods, and it also has the ability to tolerate a wide variety of substitutions in all three components.

\section{Experimental}

All chemicals were commercially available and used without further purification. Melting points were recorded on an electrothermal type 9100 melting point apparatus. The IR spectra were obtained on a 4300 Shimadzu spectrophotometer as $\mathrm{KBr}$ disks. The NMR spectra were recorded on a Bruker $250 \mathrm{MHz}$ spectrometer.

4.1. General Procedure for the Synthesis of 3,4-Dihydropyrimidin-2(1H)-Ones/Thiones. A mixture of aldehyde (1 mmol), alkyl acetoacetate $(1 \mathrm{mmol})$, urea/thiourea $(1 \mathrm{mmol})$, and trichloroacetic acid $(0.032 \mathrm{~g}, 20 \mathrm{~mol} \%)$ was stirred at $70^{\circ} \mathrm{C}$ for the appropriate time indicated in Table 1 . The progress of reactions was monitored by TLC (ethyl acetate/n-hexane). After completion of the reaction, a solid was obtained. It was allowed to cool to room temperature, and ethanol $(5 \mathrm{~mL})$ was added, and the catalyst was recovered by filtration. The filtrate was concentrated and allowed to crystallize the desired product.

\subsection{Selected Characterization Data}

Ethyl-6-Methyl-2-Oxo-4-Phenyl-1,2,3,4-Tetrahydropyrimidine5-Carboxylate. IR (KBr): 3240, 3110, 1725, 1700, 1645; ${ }^{1} \mathrm{H}$ NMR (DMSO-d6): $\delta 1.12(\mathrm{t}, J=7.5 \mathrm{~Hz}, 3 \mathrm{H}), 2.28(\mathrm{~s}, 3 \mathrm{H})$, $4.03(\mathrm{q}, J=7.5 \mathrm{~Hz}, 2 \mathrm{H}), 5.17(\mathrm{~d}, J=3.0 \mathrm{~Hz}, 1 \mathrm{H}), 7.22-7.41$ (m, 5H), 7.78 (br s, 1H), 9.22 (br s, $1 \mathrm{H})$.

Ethyl-6-Methyl-4-(4-Nitrophenyl)-2-Oxo-1,2,3,4-Tetrahydropyrimidine-5-Carboxylate. IR (KBr): 3230, 3120, 1730, 1710, $1650 ;{ }^{1} \mathrm{H}$ NMR (DMSO-d6): $\delta 1.11(\mathrm{t}, J=7.5 \mathrm{~Hz}, 3 \mathrm{H}), 2.29$ $(\mathrm{s}, 3 \mathrm{H}), 4.00(\mathrm{q}, J=7.5 \mathrm{~Hz}, 2 \mathrm{H}), 5.29(\mathrm{~d}, J=3.0 \mathrm{~Hz}, 1 \mathrm{H})$, 7.51 (d, $J=10 \mathrm{~Hz}, 2 \mathrm{H}$ ), 7.91 (br s, $1 \mathrm{H}), 8.23$ (d, $J=10.0 \mathrm{~Hz}$, 2H), 9.37 (br s, 1H).

Ethyl-6-Methyl-4-(4-Methoxyphenyl)-3,4-Dihydropyrimidin2(1H)-One-5-Carboxylate. IR (KBr): 3390, 3243, 3106, 2958, 1706, 1651,1278, 1088. ${ }^{1} \mathrm{H}$ NMR (DMSO-d6): $\delta 1.01-$ $1.20\left(\mathrm{t}, 3 \mathrm{H}, \mathrm{J}=7 \mathrm{~Hz}, \mathrm{CH}_{2} \mathrm{CH}_{3}\right), 2.30\left(\mathrm{~s}, 3 \mathrm{H}, \mathrm{CH}_{3}\right), 3.80(\mathrm{~s}$, $\left.3 \mathrm{H}, \mathrm{OCH}_{3}\right), 3.90-4.20\left(\mathrm{q}, 2 \mathrm{H}, J=7 \mathrm{~Hz}, \mathrm{CH}_{2} \mathrm{CH}_{3}\right), 5.60(\mathrm{~s}$, 1H, C4-H), 6.80-6.90 (d,2H, J = 7.2 Hz, ArH), 7.15-7.25 (d, $2 \mathrm{H}, J=7.2 \mathrm{~Hz}, \mathrm{ArH}), 7.65$ (bs, $1 \mathrm{H}, \mathrm{NH}$ ), 9.17 (bs, $1 \mathrm{H}$, $\mathrm{NH})$.

6-Methyl-4-Phenyl-3, 4-Dihydropyrimidin-2(1H)-Thione-5Carboxylate. IR (KBr): 3412, 3312, 3174, 3096, 2967, 1667,
1610, 1575. ${ }^{1} \mathrm{H}$ NMR (DMSO-d6): $\delta 1.02-1.18(\mathrm{t}, 3 \mathrm{H}, J=$ $\left.7.1 \mathrm{~Hz}, \mathrm{CH}_{2} \mathrm{CH}_{3}\right), 2.32\left(\mathrm{~s}, 3 \mathrm{H}, \mathrm{CH}_{3}\right), 4.02-4.21(\mathrm{q}, 2 \mathrm{H}, J=$ $\left.7.1 \mathrm{~Hz}, \mathrm{CH}_{2} \mathrm{CH}_{3}\right), 5.50$ (s, $\left.1 \mathrm{H}, \mathrm{C} 4-\mathrm{H}\right), 7.15-7.35$ (m, 5H, ArH), 8.90 (bs, 1H, NH), 9.95 (bs, 1H, NH).

\section{References}

[1] G. H. Mahdavinia and H. Sepehrian, "MCM-41 anchored sulfonic acid (MCM-41-R-SO $\left.{ }_{3} \mathrm{H}\right)$ : a mild, reusable and highly efficient heterogeneous catalyst for the Biginelli reaction," Chinese Chemical Letters, vol. 19, no. 12, pp. 1435-1439, 2008.

[2] C. Jiang and Q. D. You, "An efficient and solvent-free one-pot synthesis of dihydropyrimidinones under microwave irradiation," Chinese Chemical Letters, vol. 18, no. 6, pp. 647-650, 2007.

[3] Y. Yu, D. Liu, C. Liu, and G. Luo, "2007One-pot synthesis of 3,4-dihydropyrimidin-2(1H)-ones using chloroacetic acid as catalyst," Bioorganic \& Medicinal Chemistry Letters, vol. 17, no. 12 , pp. 3508-3510, 2007.

[4] S. Ghassamipour and A. R. Sardarian, "One-pot synthesis of dihydropyrimidinones by dodecylphosphonic acid as solid Bronsted acid catalyst under solvent-free conditions via Biginelli condensation," Journal of the Iranian Chemical Society, vol. 7, pp. 237-242, 2010.

[5] J. K. Joseph, S. L. Jain, and B. Sain, "Ion exchange resins as recyclable and heterogeneous solid acid catalysts for the Biginelli condensation: an improved protocol for the synthesis of 3,4-dihydropyrimidin-2-ones," Journal of Molecular Catalysis A, vol. 247, no. 1-2, pp. 99-102, 2006.

[6] M. M. Heravi, F. Derikvand, and F. F. Bamoharram, "A catalytic method for synthesis of Biginelli-type 3,4-dihydropyrimidin-2 (1H)-one using 12-tungstophosphoric acid," Journal of Molecular Catalysis A, vol. 242, no. 1-2, pp. 173-175, 2005.

[7] N. Y. Fu, Y. F. Yuan, Z. Cao, S. W. Wang, J. T. Wang, and C. Peppe, "Indium(III) bromide-catalyzed preparation of dihydropyrimidinones: improved protocol conditions for the Biginelli reaction," Tetrahedron, vol. 58, no. 24, pp. 4801-4807, 2002.

[8] N. S. Nandurkar, M. J. Bhanushali, M. D. Bhor, and B. M. Bhanage, "Y $\left(\mathrm{NO}_{3}\right)_{3} \cdot 6 \mathrm{H}_{2} \mathrm{O}$ : a novel and reusable catalyst for one pot synthesis of 3,4-dihydropyrimidin-2(1H)-ones under solvent-free conditions," Journal of Molecular Catalysis A, vol. 271, no. 1-2, pp. 14-17, 2007.

[9] C. O. Kappe, "Biologically active dihydropyrimidones of the Biginelli-type-a literature survey," European Journal of Medicinal Chemistry, vol. 35, no. 12, pp. 1043-1052, 2000.

[10] J. P. Wan and Y. Liu, "Synthesis of dihydropyrimidinones and thiones by multicomponent reactions: strategies beyond the classical Biginelli reaction," Synthesis, vol. 23, pp. 3943-3953, 2010.

[11] H. Adibi, H. A. Samimi, and M. Beygzadeh, "Iron(III) trifluoroacetate and trifluoromethanesulfonate: recyclable Lewis acid catalysts for one-pot synthesis of 3,4-dihydropyrimidinones or their sulfur analogues and 1,4-dihydropyridines via solventfree Biginelli and Hantzsch condensation protocols," Catalysis Communications, vol. 8, no. 12, pp. 2119-2124, 2007.

[12] S. D. Salim and K. G. Akamanchi, "Sulfated tungstate: an alternative, eco-friendly catalyst for Biginelli reaction," Catalysis Communications, vol. 12, no. 12, pp. 1153-1156, 2011.

[13] M. Litvic, I. Vecenaj, Z. M. Ladisic, M. Lovric, V. Vinkovic, and M. F. Litvic, "First application of hexaaquaaluminium(III) tetrafluoroborate as a mild, recyclable, non-hygroscopic acid 
catalyst in organic synthesis: a simple and efficient protocol for the multigram scale synthesis of 3,4-dihydropyrimidinones by Biginelli reaction," Tetrahedron, vol. 66, no. 19, pp. 3463-3471, 2010.

[14] M. A. Bigdeli, G. Gholami, and E. Sheikhhosseini, "P-Dodecylbenzenesulfonic acid (DBSA), a Brønsted acid-surfactant catalyst for Biginelli reaction in water and under solvent free conditions," Chinese Chemical Letters, vol. 22, no. 8, pp. 903 906, 2011.

[15] P. G. Mandhane, R. S. Joshi, D. R. Nagargoje, and C. H. Gill, "An efficient synthesis of 3,4-dihydropyrimidin-2(1H)-ones catalyzed by thiamine hydrochloride in water under ultrasound irradiation," Tetrahedron Letters, vol. 51, no. 23, pp. 3138-3140, 2010.

[16] Y. Y. Wu, Z. Chai, X. Y. Liu, G. Zhao, and S. W. Wang, "Synthesis of substituted 5-(Pyrrolidin-2-yl)tetrazoles and their application in the asymmetric Biginelli reaction," European Journal of Organic Chemistry, vol. 2009, no. 6, pp. 904-911, 2009.

[17] X. H. Chen, X. Y. Xu, H. Liu, L. F. Cun, and L. Z. Gong, "Highly enantioselective organocatalytic Biginelli reaction," Journal of the American Chemical Society, vol. 128, no. 46, pp. 1480214803, 2006.

[18] D. S. Bose, L. Fatima, and H. B. Mereyala, "Green chemistry approaches to the synthesis of 5-alkoxycarbonyl-4aryl-3,4-dihydropyrimidin-2 $(1 \mathrm{H})$-ones by a three-component coupling of one-pot condensation reaction: comparison of ethanol, water, and solvent-free conditions," Organic Chemistry, vol. 68, no. 2, pp. 587-590, 2003.

[19] J. Wannberg, D. Dallinger, C. O. Kappe, and M. Larhed, "Microwave-enhanced and metal-catalyzed functionalizations of the 4-aryl-dihydropyrimidone template," Journal of Combinatorial Chemistry, vol. 7, no. 4, pp. 574-583, 2005.

[20] K. V. N. S. Srinivas and B. Das, "Iodine catalyzed one-pot synthesis of 3,4-dihydropyrimidin-2(1H)-ones and thiones: a simple and efficient procedure for the Biginelli reaction," Synthesis, no. 13, pp. 2091-2093, 2004.

[21] I. S. Zorkun, S. Sarac, S. C. Elebi, and K. Erol, "Synthesis of 4-aryl-3,4-dihydropyrimidin-2(1H)-thione derivatives as potential calcium channel blockers," Bioorganic \& Medicinal Chemistry, vol. 14, no. 24, pp. 8582-8589, 2006.

[22] Y. Zhu, Y. Pan, and S. Huang, "Trimethylsilyl chloride: a facile and efficient reagent for one-pot synthesis of 3,4-dihydropyrimidin-2(1H)-ones," Synthetic Communications, vol. 34, pp. 3167-3174, 2004.

[23] Z. Karimi-Jaberi and M. M. ReyazoShams, "Trichloroacetic acid as a solid heterogeneous catalyst for the rapid synthesis of dihydropyrano[2,3- c ] pyrazoles under solvent-free conditions," Heterocyclic Communications, vol. 17, p. 177, 2011.

[24] Z. Karimi-Jaberi, S. Z. Abbasi, B. Pooladian, and M. Jokar, "Efficient, one-pot synthesis of tetrahydrobenzo[a]xanthen11-ones and dibenzo[a,j]xanthenes using trichloroacetic acid as a solid heterogeneous catalyst under solvent-free conditions," E-Journal of Chemistry, vol. 8, p. 1895, 2011.

[25] Z. Karimi-Jaberi and L. Zarei, "Tris(hydrogensulfato)boron catalysed rapid synthesis of 2-substituted-2,3-dihydroquinazolin-4(1H)-ones under solvent-free conditions," Journal of Chemical Research, vol. 36, pp. 194-196, 2012.

[26] Z. Karimi-Jaberi and B. Pooladian, "A facile synthesis of $\alpha, \alpha^{\prime}$ bis(substituted benzylidene) cycloalkanones catalyzed by $\mathrm{p}$ TSA under solvent-free conditions," Green Chemistry Letters and Reviews, vol. 5, pp. 187-193, 2012.
[27] Z. Karimi-Jaberi, M. Mardani, and M. Amiri, "Green, one-pot synthesis of $\alpha$-aminophosphonates catalyzed by ZnI2 at room temperature," Green Processing and Synthesis, vol. 1, pp. 191193, 2012. 


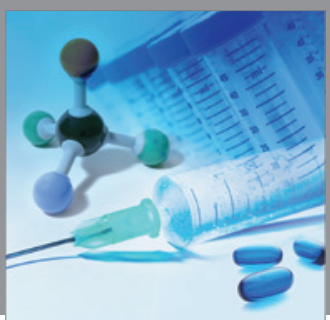

International Journal of

Medicinal Chemistry

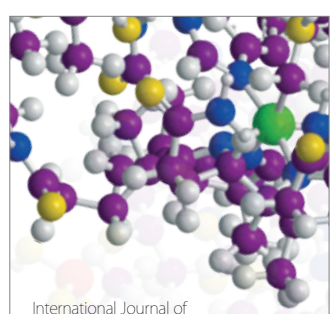

Carbohydrate Chemistry

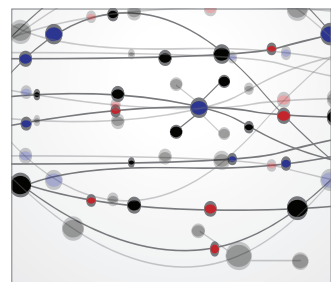

The Scientific World Journal
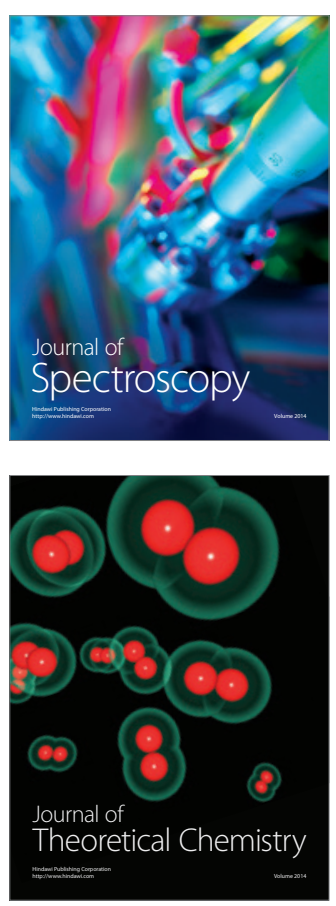
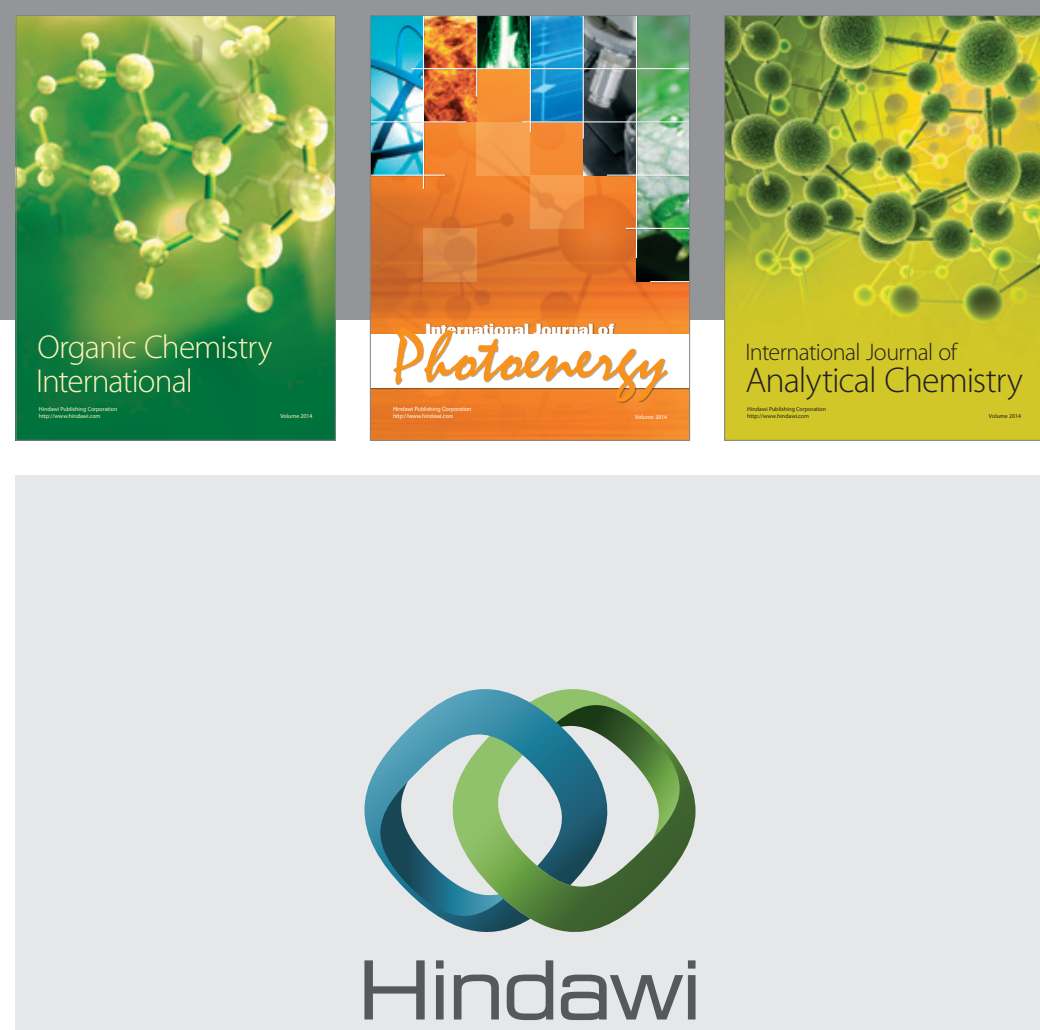

Submit your manuscripts at

http://www.hindawi.com
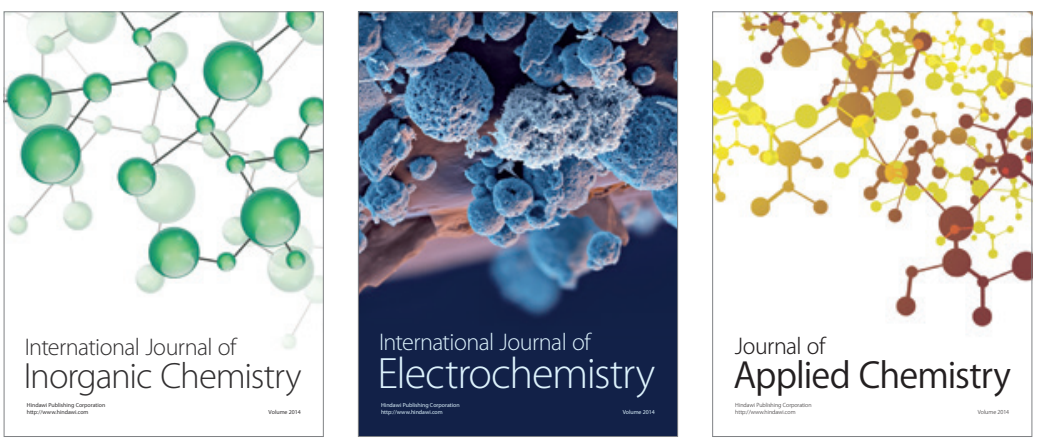

Journal of

Applied Chemistry
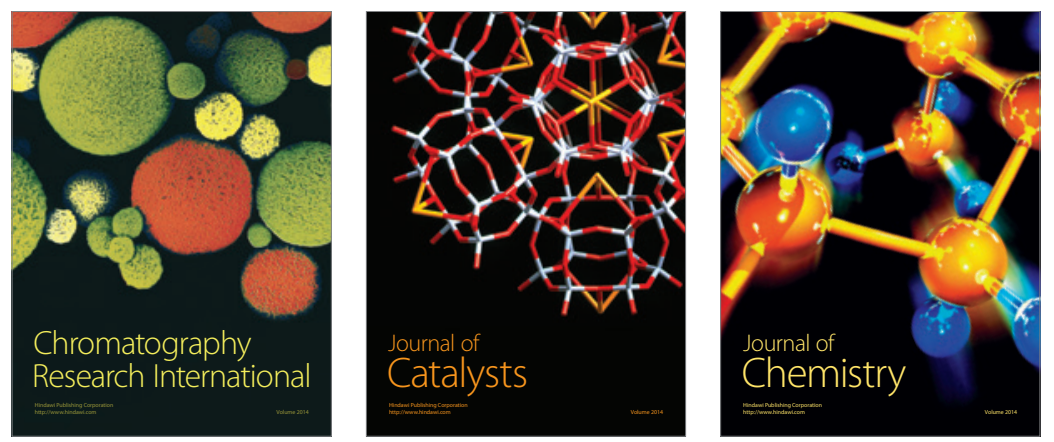
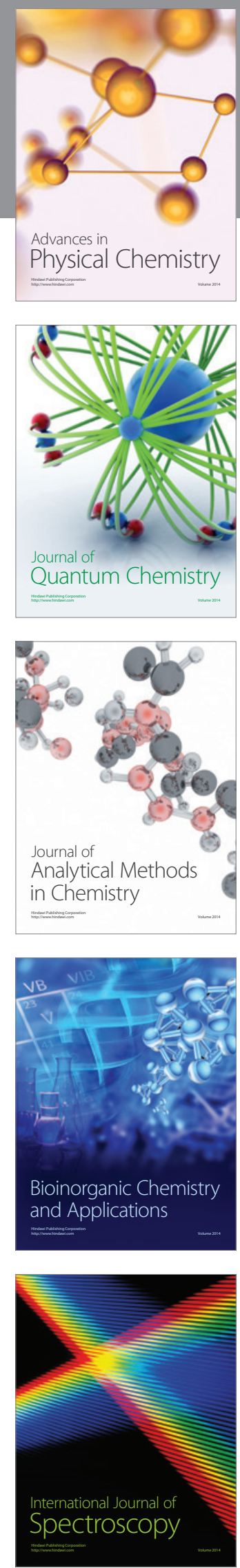